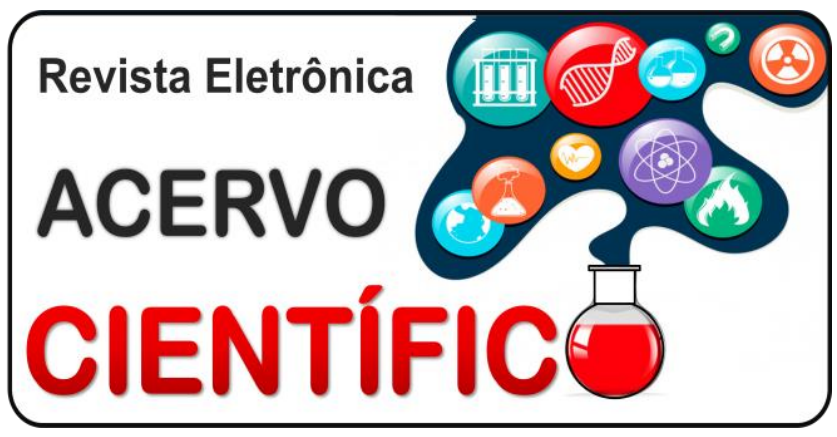

RELATO DE EXPERIÊNCIA

Recebido em: 9/2019

Aceito em: 10/2019

Publicado em: 10/2019

\title{
A monitoria acadêmica como instrumento facilitador no processo de ensino e aprendizagem no curso de enfermagem: um relato de experiência
}

\author{
Academic Monitoring as a Facilitating Instrument in the Teaching and Learning Process in \\ Nursing Course: a Report of Experience
}

\section{Monitoreo Académico como Instrumento Facilitador en el Proceso de Enseñanza y Aprendizaje en Curso de Enfermería: un Infrome de Experiencia}

Maiza Silva de Sousa ${ }^{1 *}$, Armando Sequeira Penela ${ }^{2}$, Luany Rafaele da Conceição Cruz $^{3}$, Karen Alessandra de Jesus Cuimar ${ }^{1}$, Ana Beatriz Sousa Alves ${ }^{1}$, Fernanda Tainá Oliveira da Cruz ${ }^{1}$, Taís dos Passos Sagica², Ewellyn Natália Assunção Ferreira4.

\begin{abstract}
Resumo: Esse artigo buscou descrever a experiência de uma acadêmica de enfermagem na monitoria do componente curricular Histologia Humana, evidenciando essa tarefa como um instrumento facilitador no processo de ensino e aprendizagem em uma universidade pública. Trata-se de um estudo descritivo, do tipo relato de experiência sobre as atividades desenvolvidas em sala de aula e laboratório com cerca de 40 alunos. Verificou-se que a presença do monitor em sala de aula deixava os discentes mais à vontade para levantar questionamentos sobre os conteúdos ministrados pelo docente, facilitando assim a interação entre discentes e professor orientador. Assim, a presença do monitor em sala de aula vem desmistificar a figura inquestionável atribuída ao professor, contribuindo para deixar os discentes mais à vontade, facilitar a aplicação de metodologias ativas que visam implementar o modelo horizontal de ensino e permitir uma rica troca de experiências, criando e reinventando formas de conhecer o novo.
\end{abstract}

Palavras-chave: Ensino, Tecnologia, Histologia.

\begin{abstract}
This article aimed to describe the experience of a nursing student as a Trainee Assistant in the discipline Human Histology, highlighting this task as a facilitating instrument in teaching and learning process at a public university. This is a descriptive, experience report-type study of classroom and laboratory activities with about 40 students. It was found that the presence of the Trainee Assistant in the classroom made the students more confortable to raise questions about the contents taught by the teacher, thus facilitating the interaction between students and mentor teacher. Thus, the presence of the monitor in the classroom demystifies the unquestionable figure attributed to the teacher, contributing to make students more comfortable, facilitate the application of active learning that aim to implement the horizontal model of teaching and allow a rich exchange of experiences, creating and reinventing ways to acquiring new knowledge.
\end{abstract}

Keywords: Teaching, Technology, Histology.

\footnotetext{
1 Universidade do Estado do Pará (UEPA), Belém-PA. *E-mail: maizasousa1619@gmail.com

2 Universidade Federal do Pará (UFPA), Belém-PA.

${ }^{3}$ Faculdade Integrada da Amazônia (FINAMA), Belém-PA.

4 Universidade da Amazônia (UNAMA), Belém-PA.
} 
Resumen: Este artículo tuvo como objetivo describir la experiencia de una académica de enfermería en la monitoria del componente curricular Histología Humana, evidenciando esta tarea como un instrumento facilitador en el proceso de enseñanza y aprendizaje en una universidad pública. Se trata de un estudio descriptivo, del tipo relato de experiencia sobre las actividades desarrolladas en aula y laboratorio con cerca de 40 alumnos. Se constató que la presencia del monitor en el aula dejaba a los alumnos más cómodos para plantear preguntas sobre los contenidos enseñados por el docente, facilitando así la interacción entre alumnos e profesor orientador. Así, la presencia del monitor en el aula viene a desmitificar la figura incuestionable atribuida al profesor, lo que contribuye a que los estudiantes se sientan más cómodos, facilitar la aplicación de la enseñanza orientada a la acción a la acción que pretenden implementar el modelo horizontal de enseñanza y permitir un rico intercambio de experiencias, creando y reinventando formas de conocer lo nuevo.

Palabras clave: Enseñanza, Tecnología, Histología.

\section{INTRODUÇÃO}

A monitoria acadêmica foi instituída, legalmente, a partir da Lei Federal n 5.540 de 28 de novembro de 1968. O seu artigo 41 dispõe que as universidades têm o dever de oportunizar cargos de monitoria aos alunos dos cursos de graduação. Para exercer a atividade de Monitoria Acadêmica, os alunos deverão ser submetidos a provas específicas, ter bom rendimento acadêmico e demonstrar bom desempenho técnicodidático nas atividades a serem desenvolvidas na disciplina monitorada (BRASIL, 1968).

Com isso, o monitor é capaz de auxiliar os discentes na realização das atividades propostas na disciplina, contribuindo para sua formação. De acordo com a Lei de Diretrizes e Bases da Educação Nacional n ${ }^{\circ} 9.394$ de 20 de dezembro de 1996, entre outras atribuições o monitor deve: estar presente nas aulas da disciplina, que devem ser ministradas pelo docente orientador; auxiliar o docente no desenvolvimento das atividades práticas; organizar grupos de estudos; realizar leituras complementares, afim de aprofundar os conhecimentos acerca dos conteúdos ministrados e revisá-los (BRASIL, 1996).

Assim, destaca-se que a função do monitor não se restringe a apenas sanar dúvidas dos discentes monitorados, mas também trabalhar estratégias sistematizadas, atuando como facilitador de debates e de reflexões, viabilizando os estudos em grupos e aperfeiçoando os conteúdos da disciplina, bem como os de interesse discente (FERNANDES MA, et al., 2015).

Branco Junior AG, et al. (2018) avaliou a opinião de professores orientadores sobre a importância de monitores, auxiliando no desenvolvimento da disciplina. Verificou-se que: a) 7 de 10 entrevistados citaram que o auxílio ao discente é extremamente necessário, principalmente durante as atividades em laboratório ou em sala de aula, pois é difícil orientar todos os alunos sozinho; b) 6 de 10 entrevistados citaram a sua importância na interação entre professor e alunos; c) 5 de 10 entrevistados citaram a melhora no desempenho do aluno em relação à disciplina, como fator relevante.

A partir disso, o presente estudo visa abordar a experiência de uma acadêmica de Enfermagem como Monitora do componente curricular "Histologia Humana", ministrado no $1^{\circ}$ semestre do Curso de Graduação em Enfermagem de uma universidade pública no Município de Belém-PA. Traz como enfoque a monitoria acadêmica, sendo esta visualizada como um instrumento facilitador no processo de ensino e de aprendizagem discente, assim como sua contribuição na formação acadêmica do monitor.

\section{RELATO DA EXPERIÊNCIA}

Trata-se de um estudo descritivo, do tipo relato de experiência, realizado a partir da vivência de uma acadêmica de Enfermagem na monitoria do componente curricular "Histologia Humana", do Curso de Graduação em Enfermagem da Universidade do Estado do Pará (UEPA). As atividades de monitoria foram desenvolvidas durante um ano: no $2^{\circ}$ semestre de 2017 e no $1^{\circ}$ semestre de 2018 , para as turmas do $1^{\circ}$ semestre, turno da manhã.

Cada turma era formada, em média, por 20 discentes, totalizando 40 discentes monitorados. As atividades foram desenvolvidas na sala de aula e no laboratório, onde eram realizadas as aulas teóricas, práticas e 
revisões; na biblioteca, onde nos reuníamos (na forma de grupo de estudos) para discutir as principais dificuldades encontradas em relação aos conteúdos do componente curricular; na sala de informática, onde eram repassadas orientações sobre a construção de seminários e de outros trabalhos solicitados; além de encontros alternativos no espaço da universidade, sempre que solicitado pelos discentes.

Durante o exercício da monitoria foram realizadas diversas atividades, como: acompanhar as aulas teóricas e práticas, realizadas duas vezes por semana; orientar sobre a construção dos trabalhos requisitados no decorrer do semestre, especialmente as atividades integradas em saúde (AIS), para a qual é reservado um horário de aula semanal; mediar discussões e debates sobre os conteúdos ministrados; preparar revisões e materiais de estudo complementares para os discentes, visto que encontravam dificuldade em assimilar o conteúdo prático, principalmente no início do semestre, quando se deparavam com metodologias diferenciadas em relação às utilizadas no ensino médio.

Ao acompanhar as aulas, verificou-se que a presença do monitor em sala deixava os discentes mais à vontade para levantar questionamentos sobre os conteúdos ministrados pelo docente, facilitando assim a interação entre discentes e professor orientador. No início do semestre, os discentes estavam mais tímidos e até com certo constrangimento de fazer uma pergunta que Ihes parecia tola. Porém, quando em sala de aula a monitora sempre os encorajava a fazer as perguntas, afirmando que nenhuma curiosidade é tola quando se trata de aprofundar os conhecimentos na matéria estudada.

No componente curricular de Histologia Humana percebeu-se que a contribuição da monitoria é extremamente necessária, sendo um componente curricular básico, que demanda bastante esforço e dedicação por parte dos discentes. Durante as aulas práticas no laboratório, o docente não conseguia sozinho auxiliar, simultaneamente, a todos os discentes. Com isso, a presença do monitor era imprescindível, ajudando o docente nas orientações aos discentes sobre o que observar nas lâminas, em que região procurar, além de sanar inúmeras dúvidas que fossem surgindo durante as observações. Para os conteúdos práticos foram realizadas aulas de revisão, no laboratório, para a visualização de lâminas no microscópio. Uma vez que o recurso do microscópio era inviável aos discentes em domicílio e eles precisavam visualizar as lâminas em casa, teve-se como alternativa fotografá-las e identificá-las de acordo com os tecidos e suas respectivas regiões histológicas. O recurso fotográfico ajudou bastante no estudo dos conteúdos práticos, tornando-se um método eficaz no suporte à avaliação das lâminas.

Na Figura 1 A abaixo, é evidenciado pelas setas a grande quantidade de nódulos linfoides de um baço e na Figura 1 B é observada uma das regiões do estômago, chamada fundo, onde é encontrada em grande quantidade as células parietais e as células principais. Na figura, as células principais estão identificadas pelas setas pretas e as células parietais nas setas brancas. Estas figuras foram cedidas pelo laboratório de Histologia da Universidade.

Figura 1 A e 1 B - Revisão de conteúdos com auxílio de recurso fotográfico, identificando uma porção do baço e uma região do trato gastrointestinal.

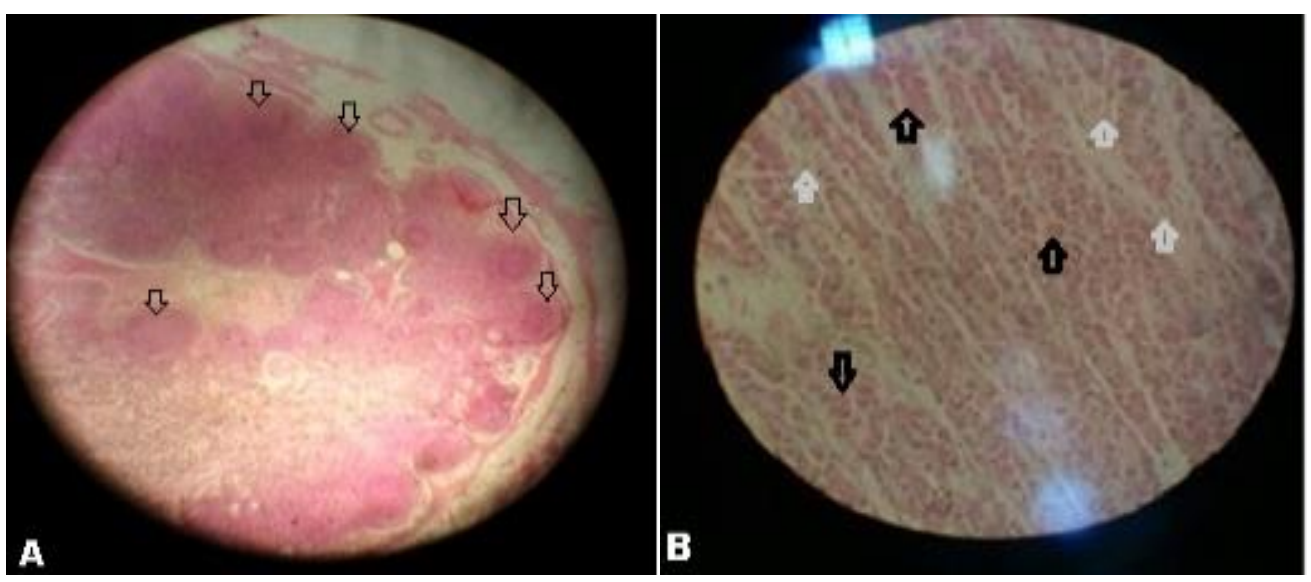

Fonte: Sousa MS, Penela AS, Cruz LRC, et al., 2018. 
Sendo assim, é inegável que a instituição dos programas de monitoria nas universidades veio facilitar o processo de ensino e de aprendizagem, sendo esta uma ferramenta extremamente necessária ao meio acadêmico. A figura do monitor tem o importante papel de mediar debates e discussões nos grupos de estudos, a fim de esclarecer as dúvidas e problematizar os assuntos abordados em sala de aula, de modo a instigar os discentes à busca das soluções, viabilizando a construção do conhecimento.

\section{DISCUSSÃO}

A melhora na interação entre discentes e docente foi um aspecto também observado por Fernandes MA, et al. (2016), em sua pesquisa sobre a influência da monitoria acadêmica no ensino e aprendizagem da Psicologia. Pois, afirma que o monitor atua como uma ponte entre os estudantes e o professor, estabelecendo relações dinâmicas e ativas, viabilizando assim a fluidez das discussões em sala.

Quanto à presença do monitor em sala de aula, Branco Junior AG, et al. (2018) verificou que $100 \%$ de sua amostra de discentes pesquisados afirmaram ser importante sua participação, visto que o monitor cumpre o importante papel de auxiliá-los durante as aulas, bem como na realização de atividades. Isto pode ser explicado, de acordo com Silveira E Sales F (2016) pelo fato de o monitor ocupar o mesmo nível acadêmico que os discentes, deixando-os mais confortáveis para questionamentos do que em relação ao docente, que supostamente estaria em um nível intelectual superior, não devendo ser questionado, na opinião dos mesmos.

As contribuições da monitoria acadêmica no processo de ensino e aprendizagem discente são inquestionáveis, possibilitando desde o acompanhamento das atividades de uma determinada disciplina até a realização de orientações e revisões dos conteúdos ministrados (FERNANDES MA, et al., 2015; LIRA MO, et al., 2015). Ainda segundo Fernandes MA, et al. (2015), cabe ao monitor desenvolver metodologias diferenciadas para facilitar o aprendizado discente, assim como também é sua função mediar grupos de estudos a fim de aprofundar os conhecimentos dos conteúdos desenvolvidos. A partir disso, verifica-se que a monitoria é um importante instrumento que facilita o processo de ensino e de aprendizagem nas universidades.

Visto que uma das atribuições do monitor é revisar conteúdos (BRASIL, 1996), foram preparados muitos materiais de revisão: principalmente dos conteúdos práticos, que eram a maior preocupação dos discentes. Foram elaborados exercícios e resumos sistemáticos dos assuntos teóricos, os quais eram disponibilizados no endereço de correio eletrônico e em mídia papel, aos discentes que desejassem.

O desempenho acadêmico dos discentes foi bastante satisfatório, visto que todos alcançaram nota superior a 8,0 pontos nas duas avaliações. Este é o valor mínimo para aprovação no componente curricular. A importância da monitoria acadêmica como fator contribuinte para o bom rendimento discente foi comprovado por Corrêa Junior JVC, et al. (2015) quando avaliaram o desempenho dos estudantes em Microbiologia dos cursos de Enfermagem e de Fisioterapia. Tais autores verificaram que, antes do estabelecimento do programa de monitoria na disciplina, o índice de reprovação foi de $42 \%$ e de $44 \%$, respectivamente. Depois de implantada a monitoria, o índice de aprovação foi de $61 \%$ e de $87 \%$.

Pode-se compreender que a monitoria acadêmica funciona como um instrumento facilitador no ensino e na aprendizagem, tanto dos discentes monitorados como do discente monitor. Isso foi demonstrado por Branco Junior AG, et al. (2016) em seu estudo sobe a contribuição da monitoria acadêmica para a tríade envolvida - Professor orientador, discentes e monitor, visto que a participação deste nas atividades de monitoria permite aprofundar os conhecimentos dos conteúdos do componente curricular, desenvolver habilidades didático-pedagógicas, influenciando na sua carreira como futuro docente, ou mesmo na criação de vínculos com os colegas de trabalho e os pacientes (PINTO MB, et al., 2016; FERNANDES J, et al., 2016).

Assim, a presença do monitor em sala de aula contribui para deixar os discentes mais à vontade e facilitar a aplicação de metodologias ativas que visam implementar o modelo horizontal de ensino na atual conjuntura educacional. Em suma, a monitoria acadêmica é uma ferramenta facilitadora na construção do conhecimento tanto para os discentes, quanto para o monitor e professor orientador, uma vez que todos participam juntos no processo de ensino e aprendizagem, numa rica troca de experiências, criando e reinventando formas de conhecer o novo e o desconhecido. 


\section{REFERÊNCIAS}

1. BRANCO JUNIOR A, et al. Monitores no processo ensino e aprendizagem: avaliação da tríade envolvida. EDUCA - Revista Multidisciplinar em Educação, 2018; 5 (10):149-164.

2. BRASIL. Ministério da Educação. Lei $n^{\circ}$ 9.394, de 20 de dezembro de 1996. Lei de Diretrizes e Bases da Educação Nacional [internet]. Diário Oficial da União, Brasília, 23 de dez, 1996.

3. BRASIL. Senado Federal, Lei Federal n 5540, de 28 de novembro de 1968.

4. CORREA JJVC, NICORENA K, STOPIGLIA CDO. A monitoria no ensino da microbiologia para cursos de fisioterapia e enfermagem na UNIPAMPA. Anais do VII Salão Internacional de Ensino, Pesquisa e Extensão Universidade Federal do Pampa, Alegrete, 2015 Anais. Rio Grande do Sul: Alegrete, 2015. 1 CD-ROM.

5. FERNANDES J, et al. Influência da monitoria acadêmica no processo de ensino e aprendizagem da psicologia. Clínica e Cultura, 2016; 2 (1): 36-43.

6. FERNANDES M, et al. Monitoria no Ensino das Paixões: acolhimento ao aluno no primeiro contato com a psicopatologia. Analytica, 2015; 4 (6): 138-150.

7. LIRA M, et al. Contribuições da monitoria acadêmica para o processo de formação inicial docente de licenciandos em Ciências Biológicas da Uepb. Il Congresso Nacional de Educação - 2015. Anais.

8. PINTO M, et al. Monitoria acadêmica: importância e contribuição para a formação do enfermeiro. Revista de Enfermagem UFPE online, 2016; 10 (6):1990-1997.

9. SILVEIRA E, SALES F. A importância do programa de monitoria no ensino de Biblioteconomia da Universidade do estado de Santa Catarina (UDESC). InCID.: Revista de Ciência da Informação e Documentação, 2016; $7(1): 131-149$. 